\title{
SCANNING AUGER MICROSCOPY AS APPLIED TO THE ANALYSIS OF HIGHLY TEXTURED YBaCu$u_{3} \mathrm{O}_{x}$ THIN FILMS*
}

\author{
ENRICO G. KEIM \\ University of T'wente, Faculty of Applied Physics, P.O. Box 217,7500 AE Enschede \\ (The Netherlands) \\ JÜRGEN HALBRITTER \\ Kerforschungszentrum Karlsruhe G.m.b.H., Postfach 3640, D.7500 Karlsruhe (F.R.G.) \\ BERND HÄUSER and HORST ROGALLA \\ University of Twente, Faculty of Applied Physics, P.O. Box 217,7500 AE Enschede \\ (The Netherlands)
}

(Received November 8, 1988)

\section{Summary}

Scanning Auger electron spectroscopy and scanning electron microscopy have been used to investigate the local composition and structure of highly textured $\hat{c}$ axis oriented $\mathrm{YBaCuO}$ films with thicknesses in the range $0.4-1 \mu \mathrm{m}$. The cuprate films were sputtered on $\mathrm{MgO}$ and sapphire (100)-oriented single-crystal substrates at room temperature followed by several anneal stages below or at $920^{\circ} \mathrm{C}$ in pure oxygen. The $\mathrm{YBaCuO} /$ sapphire sample was examined again after an additional $750^{\circ} \mathrm{C}$ air anneal for $24 \mathrm{~h}$. By applying Auger line profiling on a freshly prepared cross-sectional surface of a thin cuprate film deposited on a sapphire substrate we have been able to show that barium aluminate segregation at grain boundaries is the main cause of the higher electrical resistance usually observed for cuprate films on $\mathrm{Al}_{2} \mathrm{O}_{3}$. The (drastic) reduction in $T_{\mathrm{c}}$ can be attributed to the substitution of aluminium in the cuprate at copper sites. Severe interdiffusion has been observed for the epitaxial $\hat{c}$ axis oriented $\mathrm{YBaCu}$ oxide films grown on an $\mathrm{MgO}$ substrate, which leads to a deterioration in the superconductivity. The main reason for reduced $T_{\mathrm{c}}$ and quality of cuprate films on $\mathrm{MgO}$ is the copper loss into the substrate, the depth of penetration of copper extending more than $400 \mathrm{~nm}$ below the $\mathrm{YBaCuO}-\mathrm{MgO}$ interface. From our experimental results it is evident that Auger line profiling is an important tool in the analysis of high $T_{\mathrm{c}}$ superconducting thin films.

*Paper presented at the Symposium on High Temperature Superconductors - Preparation and Applications, at the E-MRS Fall Meeting, Strasbourg, November 8-10, 1988. 


\section{Introduction}

The development in the field of superconducting high $T_{\mathrm{c}}$ thin films tends towards improved quality of these films, driven by desires for increasing $T_{\mathrm{c}}$ and critical current density $J_{\mathrm{c}}$ and high surface quality for tunnel or SNS (superconductor-normalconductor-superconductor) junction fabrication. This has been partly achieved through changes in the preparation and the use of new materials. The role of analytical instrumentation is becoming increasingly important as all aspects of the technology associated with the production of high $T_{\mathrm{c}}$ superconducting thin films (high $T_{\mathrm{c}}$ and $\mathrm{e}_{\mathrm{c}}$ and high mechanical and chemical stability) are pushed towards their current limits.

Important aspects which still remain to be solved are intragrain weak links in the cuprate superconductor, the chemical composition and the actual structure of the cuprate film. Given the very small dimensions of the features involved, one needs an analytical technique with a very high spatial and depth resolution. Scanning Auger electron spectroscopy and angle-resolved X-ray photoelectron spectroscopy (ARXPS) are such techniques.

The objective of this paper is to show the potential of the combination of scanning Auger microscopy (SAM) and scanning electron microscopy (SEM) in clarifying the local composition and structure of highly textured $\mathrm{YBa}_{2} \mathrm{Cu}_{3} \mathrm{O}_{x}$ thin films, which have already been analysed by ARXPS [1].

Two methods have been used to characterize the superconducting cuprate films, i.e. (destructive) Auger depth profiling using argon ions, a technique which is extensively used in the depth analysis of thin films, and the recording of Auger linescans [1]. The latter technique, which we call Auger line profiling, is superior in revealing important clues to the constitution of the film resulting from interdiffusion, growth and oxygen annealing and, in particular, precipitation and enhanced diffusion at grain boundaries. It does not suffer from sputter artefacts and serious charging problems (on reaching the cuprate insulator interface) often encountered in destructive Auger depth profiling [2]. The SAM-SEM measurements have been performed using the PHI 600 scanning Auger microprobe, the achieved lateral resolution being $20 \mathrm{~nm}$. The cuprate films were deposited on $\langle 100\rangle$-oriented $\mathrm{MgO}$ and sapphire single crystalline substrates by r.f. magnetron sputtering from a stoichiometric sinter target in a turbopumped vacuum chamber, followed by several anneal stages in $\mathrm{O}_{2}$ up to $920^{\circ} \mathrm{C}$ [3]. The cuprate film on the sapphire substrate received after the ARXPS measurement an additional air anneal at $750{ }^{\circ} \mathrm{C}$ for $24 \mathrm{~h}$.

The elemental Auger linescans were recorded on a freshly prepared cross-sectional surface of a thin film deposited on a substrate and fractured in air. The following experimental conditions were used: primary beam energy $E_{\mathrm{p}}=15 \mathrm{keV}$; primary beam current $I_{\mathrm{p}}=0.25 \mathrm{nA} ; 160$ points per line; analysis time per point $\tau=5 \mathrm{~s}$; energy resolution $\Delta E / E$ of the cylindrical mirror analyser, $1.2 \%$; additional point analyses below the cuprate-substrate interface using $\tau=40 \mathrm{~s}$. Under these conditions the detection limit for copper is less than $3 \%$ (atomic concentration) [4]. The lateral resolution, defined as the 
distance between two points on the Auger linescan corresponding to $20 \%$ and $80 \%$ of the maximum Auger signal, was found to be about $80 \mathrm{~nm}$. The linescans have been corrected for topographic effects. For the experimental details concerning the Auger sputter profiles we refer to ref. 1.

Our SAM results are in good agreement with ARXPS analyses [5] performed on the same samples discussed in the present paper. ARXPS has been most useful in identifying the chemical signature of the reaction cinder, i.e. the initial layer of the cuprate film with a thickness less than $10 \mathrm{~nm}$. Scanning Auger electron spectroscopy has yielded significant complementary information [1]. The cuprate films analysed here are state of the art for July 1987, i.e. not optimized for superconducting application but excellent for the illustration of the power of Auger line profiling in clarifying diffusion, interface layers and deterioration.

\section{Problem}

A thin film consisting of yttrium, barium, copper and oxygen has been prepared according to the recipe described in ref. 3 with sapphire as the substrate. After the measurement of the film properties an extra $750 \mathrm{C}$ air anneal for $24 \mathrm{~h}$ was applied resulting in a deterioration in $T_{\mathrm{c}}$ and $J_{\mathrm{c}}$ [1]. Moreover, ARXPS measurements showed an enrichment of $\mathrm{BaO}_{2}$ in the surface layer [5]. The question arose as to which factors caused the reduction in $T_{\text {c }}$ and the higher electrical resistance of $\mathrm{YBaCuO}$ films on $\mathrm{Al}_{2} \mathrm{O}_{3}$.

\section{Analysis results}

Figure 1(a) shows the SEM image of a freshly prepared cross-sectional surface of a thin $\mathrm{YBaCuO}$ film on a sapphire substrate and fractured under atmospheric conditions. The Auger linescans in Fig. 1(b) display the topographically corrected elemental Auger intensity across the interface in the SEM image (Fig. 1(a)) indicated by the arrows. As observed in the Auger $\left(\Lambda r^{+}\right)$sputter profile [1], a significant enrichment of barium (relative atomic concentration) occurs both at the outer surface (about $0.12 \mu \mathrm{m}$ ) and near the cuprate-sapphire interface $(0.4 \mu \mathrm{m})$. The locally enhanced aluminium intensity at $0.12 \mu \mathrm{m}$ is due to preferential segregation of aluminium at grain boundaries, as will be shown in Fig. 2(b). Only between 0.15 and $0.40 \mu \mathrm{m}$ are the barium and copper intensities and the yttrium and oxygen intensities (not shown) constant, which implies a homogeneous cuprate layer of about $0.25 \mu \mathrm{m}$. The drop in elemental Auger intensities between 0 and $0.1 \mu \mathrm{m}$ is due to carbon contamination of the freshly prepared surface by fracturing in air [1]. The relatively high barium concentration in the surface region is due to the formation of the thermodynamically stable $\mathrm{BaO}_{2}$ [5], forming crystallites as shown in ref. 1 by the elemental Auger mappings. Furthermore, the build-up of barium is enhanced by the rapid barium diffusion in oxygen- 

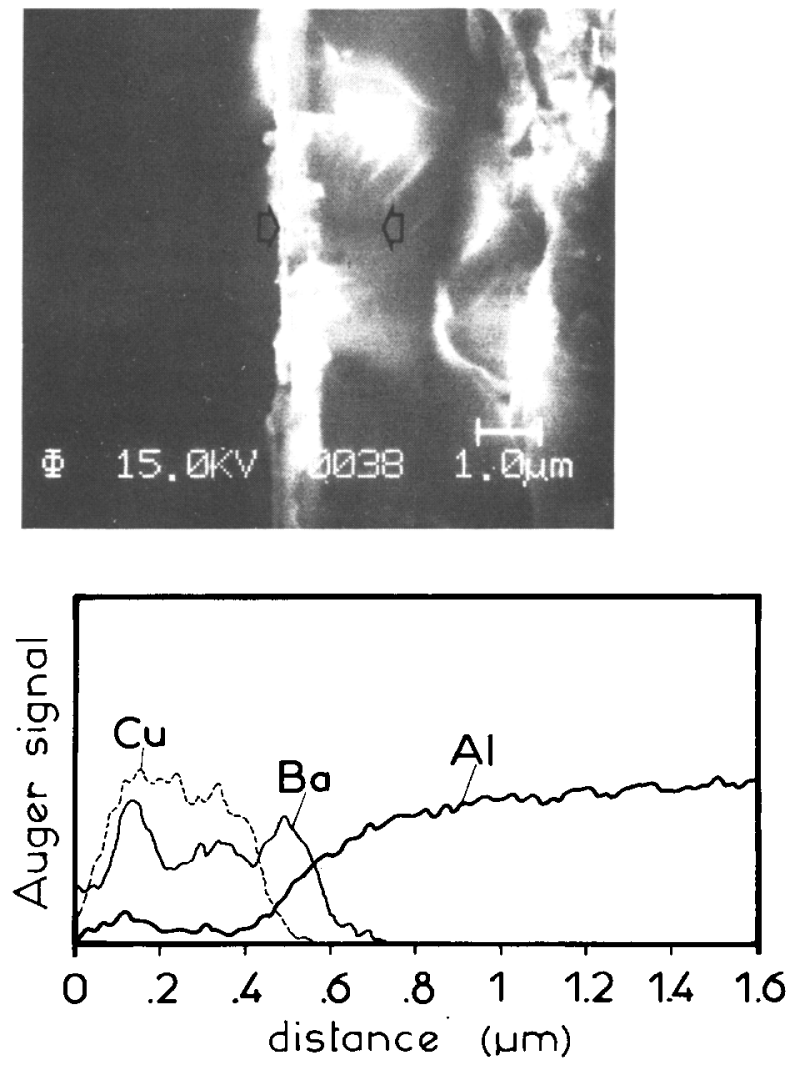

Fig. 1. (a) SEM image of a fractured sapphire substrate surface with a YBaCuO film on top. (Magnification, $10000 \times$ ). (b) Auger elemental linescaiss, recorded between the two arrows in the SEM image of (a).

deficient cuprate via the oxygen vacancies in the copper chains [6]. Near the cuprate-sapphire interface the increase in barium strongly suggests the formation of the thermodynamically stable barium aluminate [7]. Supporting evidence is given by the linescans which were recorded parallel to the substrate in the film at about $0.15 \mu \mathrm{m}$ below the surface of the top layer (SEM image in Fig. 2(a)). The linescans displayed in Fig. 2(b) show only an enrichment of aluminium, barium and oxygen at a grain boundary (Fig. 2(a)) which, in our opinion, is an indication of the insulating $\mathrm{BaAl}_{2} \mathrm{O}_{4}$. The barium aluminate segregation at grain boundaries is likely to be the reason for the higher electrical resistance of films on $\mathrm{Al}_{2} \mathrm{O}_{3}$. Furthermore, aluminium is substituted in the cuprate at copper sites (Fig. 1(b) and ref. 1) thus explaining the $T_{\mathrm{c}}$ reductions [8].

In contrast to sapphire substrates, in $\mathrm{MgO}$ substrates an enhanced concentration of barium below the cuprate film has not been observed, neither from the sputter profiles [1] nor from the Auger line profiles. Figure 


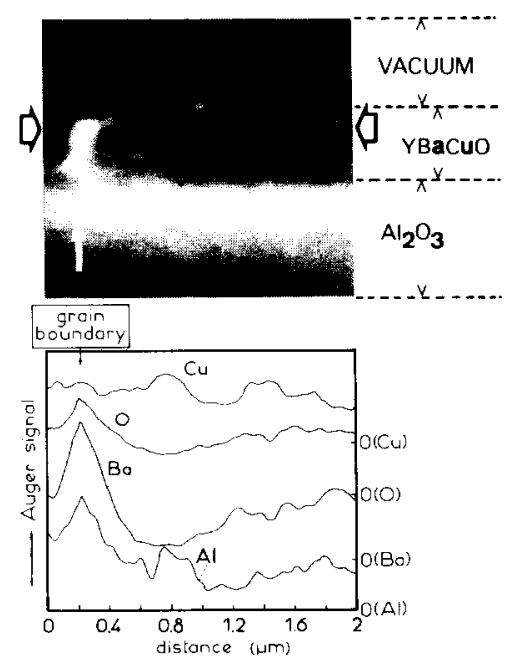

Fig. 2. (a) SEM image of a fractured sapphire substrate surface with a YBaCuO film on top. (Magnification, $25000 \times$ ). (b) Auger elemental linescans, recorded between the two arrows in the $\mathrm{SEM}$ image of (a), in the $\mathrm{YBaCuO}$ film parallel to the substrate. The vertical axis on the right-hand side shows the level of zero Auger intensity for each element.

3(a) shows the SEM image of a fractured MgO substrate surface with a $\mathrm{YBaCuO}$ film on top. The sample did not receive a prolonged air anneal as in the case of the $\mathrm{YBaCuO} /$ sapphire sample and we did not find any indication for a more stable $\mathrm{Mg}-\mathrm{Cu}-\mathrm{O}$ compound. Displayed in Fig. 3(b) are the Auger line profiles for copper and magnesium recorded between the arrows in the SEM image of Fig. 3(a). Form the linescan it has heen determined that the depth of penetration of copper extends more than $400 \mathrm{~nm}$ below the $\mathrm{YBaCuO}-$ $\mathrm{MgO}$ interface, in agreement with our earlier preliminary copper Auger linescan [9]. As can be seen from the line profile in Fig. 3(b), the main reason for reduced $T_{\mathrm{c}}$ and quality of cuprate films on $\mathrm{MgO}$ is the copper loss into the $\mathrm{MgO}$ substrate. This on the one hand allows for wetting and oriented growth in sputter deposition [9] but on the other hand yields a copper loss, degrading the quality of the films. This is prevented in part by copper doping of $\mathrm{MgO}$ single crystals and by reduced annealing temperatures and times as shown by the currently prepared films. We investigated a number of $\mathrm{YBaCuO}-\mathrm{on}-\mathrm{MgO}$ samples, but segregation to grain boundaries or interfaces, however, could not be observed.

\section{Conclusion}

The present study shows that Auger line profiling is very useful in clarifying the local composition and structure of highly textured $\mathrm{YBaCuO}$ 

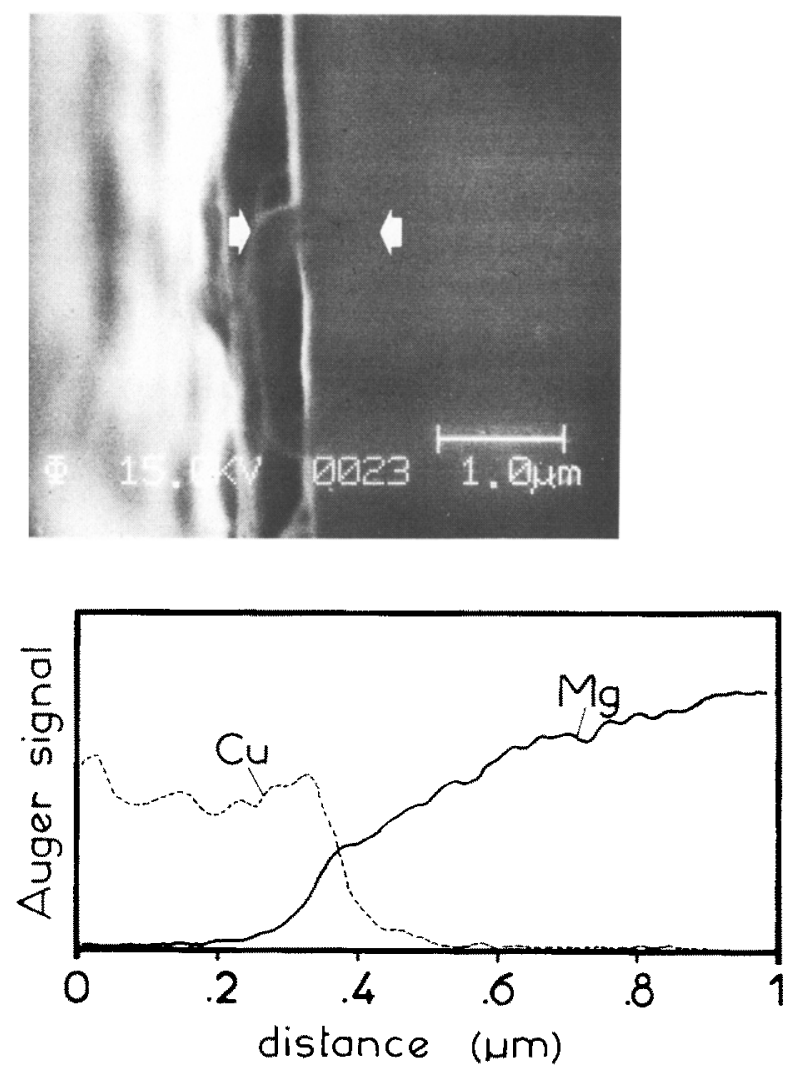

Fig. 3. (a) SEM image of a fractured $\mathrm{MgO}$ substrate surface with a YBaCuO film on top. (Magnification, $20000 \times$ ). (b) Auger line profiles for copper and magnesium recorded between the two arrows in the SEM image of (a).

thin films with a thickness of $0.4-1 \mu \mathrm{m}$. Auger line profiling gives essentially the same results as destructive Auger sputter profiling but without any sputter artefacts, however, such as preferential sputtering and induced surface roughening, and serious charging problems. Moreover, the determination of the film thickness is very easily carried out just by observing the material contrast in the SEM image of the fractured surface. Our future Auger line profiling measurements will be performed on samples which are fractured in situ in ultrahigh vacuum, thus avoiding carbon contamination on the freshly prepared surface. From our extensive set of data (refs. 1, 5 and 9, and this work) we may speculate that the high depth resolution of ARXPS. yielding the chemical signature of the surface region (thickness less than $10 \mathrm{~nm}$ ), and the high lateral resolution of Auger line profiling at substrates, electrical contacts or grain boundaries, will show directions in the future development of new high(er) $T_{\mathrm{c}}$ superconducting films. 


\section{References}

1 J. Halbritter, B. Häuser, E. G. Keim, H.-J. Mathes, P. Walk and H. Rogalla, Proc. ASC Conf., San Francisco, CA, 1988, IEEE Trans. Magn., to be published.

2 S. Hofmann, in D. Briggs and M. P. Seah (eds.), Practical Surface Analysis by Auger and X-ray Photoelectron Spectroscopy, Wiley, Chichester, 1985, p. 141.

3 B. Häuser and H. Rogalla, in S. A. Wolf and V. Kresin (eds.), Novel Superconductivity, Plenum, New York, 1987, p. 951.

4 Detectability limits for Auger analysis, PHI Techn. Bull. T8401, May 5, 1984 (Physical Electronics Industries, Perkin-Elmer).

5 J. Halbritter, B. Häuser, H.-J. Mathes, H. Rogalla and P. Walk, Physica C, 153 (1988) 127; Z. Phys. B, 73 (1988) 277.

6 L. D. Marks, D. J. Li, H. Shivahara and J. P. Zhang, J. Electron Micros. Tech. 8 (1988) 297.

7 T. Yamashita, A. Kawekani, S. Noge, M. Tekate, T. T. Komatsu and K. Matusite, Abstracts FED HiT .Sc Workshop, Miyagi-Zao, Japan, June 1988, Research and Development Assuciation for Future Devices, Tokyo, p. 161.

8 A. Matsushita, H. Aoki, Y. Asada, T. Hatano, K. Kimura, T. Matsumoto, K. Nakamura and K. Ogawa, Physica B, 148 (1987) 342.

9 B. Häuser, E. G. Keim, H. Rogalla and A. van Silfhout, Appl. Phys. A, 46 (1988) 339. 